\title{
EDITORIAL
}

\section{VISIBILIDAD CIENTÍFICA: desafíos enfrentados y logros alcanzados por IPSA Scientia en tiempos de pandemia}

Nuestra apuesta este año 2020, con la implementación del sistema de gestión editorial OJS y el lanzamiento del volumen 5 que hoy presentamos, no fue otra sino el logro de mayor visibilidad de IPSA Scientia. Emprendimos el andar con mucho entusiasmo y motivación, con la mayor de las voluntades en un gigante esfuerzo ante las circunstancias que afectaron al planeta entero tras la inesperada irrupción de un actor que transformó la realidad, un intruso que cambió el significado de la normalidad, un nuevo integrante de la sociedad con el que debemos saber convivir y por el cual aprendimos a redimensionar el valor de la vida familiar.

En este escenario, gracias al sentido de pertenencia de quienes hacemos parte de este equipo, vimos cristalizar el esfuerzo en logros que nos llenan de profunda satisfacción: el registro en por lo menos diez repositorios, índices y bases de datos; la difusión en línea de nuestros contenidos tras una presencia en formato impreso durante cuatro años; la constitución de un equipo editorial internacional comprometido; la acogida de nuestra propuesta por parte de la comunidad científica latinoamericana del que hacen parte un grupo de investigadores que nos honran con sus contribuciones en este número.

Comprendemos que esto apenas es el principio, y que la visibilidad científica no está del todo consolidada, pero existe el compromiso por no abandonar el camino. Con el uso de números DOI para la identificación de artículos en acceso abierto y el tratamiento de metadatos; la difusión apoyada en canales social media; y una gestión editorial transparente impulsada por métricas de analítica de datos, sumamos intenciones con miras a mayores garantías de calidad científica.

Es esta la plataforma que sostiene al volumen 5 de la revista IPSA Scientia, organizado en un cuerpo de dieciocho contribuciones, que abordan temas desde diferentes ópticas, disciplinas y latitudes. Inicia con el trabajo de Geizzelez, quien desde Venezuela nos presenta un Modelado del registro automatizado de la gestión docente para una institución universitaria a partir de la definición de requerimientos que orientaron el diseño del modelo de datos respectivo.

Seguidamente, los autores Fong, Severiche y Fong realizan un análisis de los Sistemas de acondicionamiento de aire y ventilación como foco infeccioso de SARS CoV-2 en hospitales, clínicas e instituciones de salud colombianas, para explicar la necesidad de utilizar filtros de alta eficiencia HEPA y cámaras germicidas con luz ultravioleta. Continuando con el tema preventivo ante la pandemia, el trabajo realizado por Palacio titulado Nistatina como inhibidor de caveolas: tratamiento preventivo para el contagio por COVID-19, cuyo propósito fue formular la hipótesis 
de administrar un medicamento para prevenir la enfermedad por contagio de coronavirus basado en el uso de nistatina bajo la presentación de suspensión oral.

Por otro lado, Cohen, Linares y Briceño nos ofrecen el trabajo Caracterización de la cultura innovativa en la cooperación universidad-empresa del departamento del Magdalena, Colombia; cuyos hallazgos demuestran la presencia de las condiciones adecuadas para apalancar estrategias de implementación de ecosistemas de innovación que coadyuven a la cooperación intersectorial y la articulación entre los actores sociales involucrados, desde la perspectiva de la cultura innovativa en la universidad.

Por su parte, Quintero y Cárdenas establecen los Tipos de marketing en comercios textiles de la Guajira Colombiana, destacando que los tipos social, comercial y de causa generar rentabilidad de ventas y satisfacción del cliente. Mientras que Patiño nos presenta su artículo titulado Crosslinked carboxymethyl cellulose-SiO ${ }_{2}$ hidrogels fabrication: coposition and termal stability towards biomedical applications, cuyos resultados sugieren un adecuado proceso de fabricación de hidrogeles entrecruzados de $\mathrm{CMC}-\mathrm{SiO}_{2}$ como material novedoso con propiedades fisicoquímicas prometedoras.

También es relevante la contribución de Campo, Ceballos y Herazo, con su investigación Denominaciones para trastornos mentales conocidas por estudiantes de medicina: un estudio cualitativo, que busca explorar las denominaciones conocidas para trastornos mentales en un grupo de estudiantes de medicina de Santa Marta, Colombia. Y por otro lado, González analiza las evidencias que permiten asociar las afectaciones en la piel de las personas y el contagio por COVID-19, tras una revisión bibliográfica realizada como parte de la investigación titulada COVID-19 en Iberoamérica: Un acercamiento desde la dermatología.

Asimismo, desde Venezuela compartimos el trabajo de Nava relativo al Sistema de benchmarking como estrategia de innovación tecnológica en el sector crudos pesados y extrapesados, en el que a través del cuadro de mando integral se llevó a cabo un plan estratégico para consolidar la innovación tecnológica en el sector. En otro orden de ideas, se tiene el estudio de Vega, Ceballos y Pardo acerca de los Riesgos psicosociales en trabajadores calificados por enfermedad laboral frente a trabajadores que esperan por calificación, con el objetivo de establecer las diferencias entre los riesgos psicosociales entre estos dos círculos de trabajadores comparados.

Otra contribución de interés es la investigación de García y Mogollón, quienes nos hablan de la Gamificación con procesos cognitivos para mejorar niveles de comprensión lectora en estudiantes de octavo grado, mediante la aplicación del entorno gamificado llamado "Mundo Awen" para el fortalecimiento de los procesos cognitivos y de comprensión lectora. Por su parte, Montoya, Robayo y Monroy nos presentan su Evaluación de la fatiga laboral en conductores de la Cooperativa de Transporte del municipio de Planadas, en Colombia, donde se aborda las condiciones que generan fatiga laboral para documentar los agentes etiológicos y los factores de riesgo que se presentan en dicha cooperativa.

Para cerrar con el grupo de estudios centrados en el tema de la pandemia, González, Ustáriz y Cadavid nos exponen el Uso de mascarillas en tiempos de COVID-19: algunas manifestaciones

IPSA SCIENTIA - Vol. 5 No $1-2020$ 
en la piel del personal de la salud, trabajo en el que se plantean una serie de medidas para reducir las lesiones por presión relacionadas con dispositivos médicos, en combinación con fricción y cizallamiento.

La apertura internacional de IPSA Scientia nos lleva hasta Argentina, donde De Vicenzi, Marcano y Macri nos exponen su postura acerca de La práctica educativa bajo el lente de la teoría de la actividad, y concluyen que el método elaborado abonan a la construcción de esquemas de investigación para el estudio de las buenas prácticas para la calidad formativa. También el trabajo de Mejía titulado Políticas públicas en Ciencia, Tecnología e Innovación: el caso del ICBF Regional Atlántico, Colombia, cuyos resultados evidencian que los servicios institucionales del ICBF se encuentran alineados con los Programas de Desarrollo vigentes y la política pública colombiana en CTI.

Un estudio colaborativo entre investigadores de Ecuador y Cuba titulado Incidencias de las contradicciones sociales en el comportamiento del consumidor turístico del Ecuador, es presentado por Lemoine, Fernández y Villacís, quienes con su aporte destacan la necesidad de adoptar un enfoque integrado basado en las dimensiones económicas, sociales y ambientales del destino, para lograr una gestión eficiente y eficaz del mismo. Seguidamente, Gutiérrez nos ofrece su Caracterización de la gerencia en la deserción estudiantil en instituciones de educación superior del Atlántico, Colombia, cuyos hallazgos evidencian las deficientes competencias en habilidades gerenciales ante el problema de la deserción estudiantil; siendo los factores del mismo determinados por aspectos individuales, socioeconómicos, académicos e institucionales; y finalmente tipificarla por cohorte, período y ausencia intersemestral.

Finalmente, presentamos el trabajo de Fong, Fong y Colpas, titulada Autoeficacia y su asociación con la autoestima, motivación intrínseca y nivel educativo de la madre en estudiantes universitarios, con el objetivo de estudiar la correlación entre la autoeficacia y la autoestima, motivación intrínseca y nivel educativo de la madre en estudiantes universitarios de ingeniería de la Universidad de Cartagena, mostrando significancia estadística entre autoeficacia, motivación intrínseca y nivel educativo de la madre, y ninguna con la autoestima.

Fue un año de retos y dificultades, que supimos afrontar con disposición y voluntad. En nombre del equipo editorial, quiero agradecer a este grupo de investigadores que nos acompañan desde diferentes latitudes del continente: Colombia, Venezuela, Argentina, Ecuador y Cuba, por contribuir con el desarrollo de la ciencia desde esta palestra: nuestro compromiso sigue latente y con la firme convicción de seguir adelante en la nueva normalidad que nos depara los tiempos por venir.

PhD. José Alexander Linares Morales

Editor

IPSA SCIENTIA - Vol. 5 No $1-2020$ 